\title{
The hot subdwarf B + white dwarf binary KPD 1930+2752
}

\section{A supernova type la progenitor candidate ${ }^{\star, \star \star, \star \star \star \star, \dagger}$}

\author{
S. Geier ${ }^{1}$, S. Nesslinger ${ }^{1}$, U. Heber ${ }^{1}$, N. Przybilla ${ }^{1}$, R. Napiwotzki ${ }^{2}$, and R.-P. Kudritzki ${ }^{3}$ \\ 1 Dr.-Remeis-Sternwarte, Institute for Astronomy, University Erlangen-Nuremberg, Sternwartstr. 7, 96049 Bamberg, Germany \\ e-mail: geier@sternwarte.uni-erlangen.de \\ 2 Centre of Astrophysics Research, University of Hertfordshire, College Lane, Hatfield AL10 9AB, UK \\ 3 Institute for Astronomy, University of Hawaii, 2680 Woodlawn Drive, Honolulu, HI 96822, USA
}

Received 25 July 2006 / Accepted 27 October 2006

\section{ABSTRACT}

\begin{abstract}
Context. The nature of the progenitors of type Ia supernovae is still under debate. KPD 1930+2752 is one of the best SN Ia progenitor candidates known today. The object is a double degenerate system consisting of a subluminous B star (sdB) and a massive white dwarf (WD). Maxted et al. (2000) conclude that the system mass exceeds the Chandrasekhar mass. This conclusion, however, rests on the assumption that the sdB mass is $0.5 M_{\odot}$. However, recent binary population synthesis calculations suggest that the mass of an sdB star may range from $0.3 M_{\odot}$ to more than $0.7 M_{\odot}$.

Aims. It is therefore important to measure the mass of the sdB star simultaneously with that of the white dwarf. Since the rotation of the sdB star is tidally locked to the orbit, the inclination of the system can be constrained if the sdB radius and the projected rotational velocity can be measured with high precision. An analysis of the ellipsoidal variations in the light curve allows the constraints derived from spectroscopy to be tightened.

Methods. We derived the mass-radius relation for the sdB star from a quantitative spectral analysis of 150 low-resolution spectra obtained with the Calar Alto $2.2 \mathrm{~m}$ telescope using metal-rich, line-blanketed LTE model atmospheres with and without NLTE line formation. The projected rotational velocity was determined for the first time from 200 high-resolution spectra obtained with the Keck I $10 \mathrm{~m}$ and with the ESO-VLT $8.2 \mathrm{~m}$ telescopes. In addition a reanalysis of the published light curve was performed.

Results. The atmospheric and orbital parameters were measured with unprecedented accuracy. In particular the projected rotational velocity $v_{\mathrm{rot}} \sin i=92.3 \pm 1.5 \mathrm{~km} \mathrm{~s}^{-1}$ was determined. Assuming the companion to be a white dwarf, the mass of the sdB is limited between $0.45 M_{\odot}$ and $0.64 M_{\odot}$ and the corresponding total mass of the system ranges from $1.33 M_{\odot}$ to $2.04 M_{\odot}$. This constrains the inclination to $i>68^{\circ}$. The photometric analysis allows the parameters to be constrained even more. A neutron star companion can be ruled out and the mass of the sdB is limited to the range between $0.45 M_{\odot}$ and $0.52 M_{\odot}$. The total mass of the system ranges from $1.36 M_{\odot}$ to $1.48 M_{\odot}$ and hence is likely to exceed the Chandrasekhar mass. The inclination angle is $80^{\circ}$ and the light curve shows weak and shallow signs of eclipses. A high-precision light curve is needed in order to accurately measure these eclipses. So KPD 1930+2752 qualifies as an excellent double degenerate supernova Ia progenitor candidate.
\end{abstract}

Conclusions.

Key words. binaries: spectroscopic - stars: atmospheres - stars: individual: KPD 1930+2752 - subdwarfs - supernovae: general

\section{Introduction}

Supernovae of type Ia (SN Ia) play an important role in the study of cosmic evolution. They are regarded as the best standard candles for determining the cosmological parameters $H_{0}, \Omega$, and $\Lambda$ (e.g. Riess et al. 1998; Leibundgut 2001;

^ Some of the data presented here were obtained at the W.M. Keck Observatory, which is operated as a scientific partnership among the California Institute of Technology, the University of California, and the National Aeronautics and Space Administration. The Observatory was made possible by the generous financial support of the W.M. Keck Foundation.

$\star \star$ Based on observations collected at the Centro Astronómico Hispano Alemán (CAHA) at Calar Alto, operated jointly by the MaxPlanck Institut für Astronomie and the Instituto de Astrofísica de Andalucía (CSIC).

$\star \star \star$ Based on observations at the Paranal Observatory of the European Southern Observatory for programme 167.D-0407.

$\dagger$ R. N. gratefully acknowledges support from a PPARC Advanced Fellowship.
Perlmutter et al. 1999), together with the WMAP probe for anisotropies in the cosmic background radiation (e. g. Bennett et al. 2003). Although some of the most important discoveries in modern cosmology, in particular the accelerated expansion of the universe, were initially derived from distance measurements of SN Ia with high redshifts, the nature of their progenitors is still under debate (Livio 2000). The progenitor population is crucial information for backing the assumption that distant SN Ia can be used as standard candles like the ones in the local universe.

There is general consensus that only the thermonuclear explosion of a white dwarf (WD) is compatible with the observed features of SN Ia. For this a white dwarf has to accrete mass from a close companion to reach the Chandrasekhar limit of $1.4 M_{\odot}$ (Hamada \& Salpeter 1961). Two main scenarios of mass transfer are currently under discussion. In the so-called single degenerate (SD) scenario (Whelan \& Iben 1973), the massdonating component is a red giant/subgiant, which fills its Roche lobe and is continually transferring mass to the white dwarf. According to the so-called double degenerate (DD) scenario 
(Iben \& Tutukov 1984), the mass-donating companion is a white dwarf, which eventually merges with the primary due to orbital shrinkage caused by gravitational wave radiation.

A progenitor candidate for the DD scenario must have a total mass near or above the Chandrasekhar limit and has to merge in less than a Hubble time. Systematic radial velocity (RV) searches for DDs have been undertaken (e.g. Napiwotzki 2003, and references therein). The largest of these projects was the ESO SN Ia Progenitor Survey (SPY). More than 1000 WDs were checked for RV-variations (e.g. Napiwotzki et al. 2003). SPY detected $\sim 100$ new DDs (only 18 were known before). One of them may fulfill the criteria for progenitor candidates, even though the error margins are quite high (Napiwotzki et al. 2005; Karl 2004).

KPD 1930+2752 was identified as a subdwarf B (sdB) star in the Kitt Peak - Downes survey (Downes 1986). The parameters that were derived from spectroscopy by model atmosphere fits (Saffer \& Liebert 1995) are consistent with the theoretical instability strip predicted by Charpinet et al. (1996). After the first pulsating sdB stars with short periods (EC 14026 stars) were discovered (Kilkenny et al. 1997; Koen et al. 1997; Stobie et al. 1997), Billères et al. (2000) initiated a survey to search for similar objects in the northern hemisphere. KPD 1930+2752 was selected from the list of Saffer \& Liebert (1995) for their fast photometry program. They detected multiperiodic variations with short periods and low amplitudes. In addition to $44 p$-mode pulsations they found a strong variation at a much longer period of about $4100 \mathrm{~s}$. This variation could be identified as an ellipsoidal deformation of the sdB star most likely caused by a massive companion. Billères et al. (2000) predict the period of the binary to be two times the period of this brightness variation $(P=8217.8 \mathrm{~s}=0.095111 \mathrm{~d})$.

This was proven by Maxted et al. (2000), who measured a radial velocity curve of KPD $1930+2752$ that matched the proper period. The radial velocity amplitude $K=349.3 \pm$ $2.7 \mathrm{~km} \mathrm{~s}^{-1}$, combined with an assumption of the canonical mass for $\mathrm{sdB}$ stars $M_{\mathrm{sdB}}=0.5 M_{\odot}$, led to a lower limit for the mass of the system derived from the mass function. This lower limit $M \geq 1.47 M_{\odot}$ exceeded the Chandrasekhar mass of $1.4 M_{\odot}$ (Hamada \& Salpeter 1961). Because there was no sign of a companion in the spectra, it was concluded that the unseen object must be a white dwarf. The system is considered to become DD when the subdwarf eventually evolves to a white dwarf. Orbital shrinkage caused by gravitational wave radiation will lead to a merger of the binary in about $200 \mathrm{Myr}$, which is much less than a Hubble time. Combining all the evidence, Maxted et al. conclude that KPD $1930+2752$ could be a good candidate for the progenitor of a Type Ia supernova.

From the theoretical point of view, Ergma et al. (2001) questioned the DD scenario in the case of KPD 1930+2752. Their simulations based on the derived parameters of Maxted et al. (2000) suggest that the merger will lead to a single massive ONeMg white dwarf rather than a supernova explosion. The merger of two white dwarfs is a rather complicated process compared to slow accretion of material onto a heavy white dwarf. Detailed merger simulations in 3D are not yet avaible, but urgently needed to learn more about this possible SN Ia progenitor channel.

The subluminous B stars form the hot end of the horizontal branch, the so called extreme horizontal branch (EHB, Heber 1986). The EHB models imply that they are core helium-burning objects with tiny hydrogen dominated envelopes, too thin to sustain hydrogen burning. The core mass is fixed by the onset of the core helium flash at the tip of the red giant branch and only depends slightly on metallicity and helium abundance; hence, the canonical core mass is restricted to a very narrow range of 0.46 to $0.5 M_{\odot}$. Unlike normal horizontal branch stars, EHB stars do not ascend the asymptotic giant branch (AGB) after core helium burning has terminated, but evolve to higher temperatures until the white dwarf cooling sequence is reached.

Binary evolution scenarios have been proposed (e.g. Han et al. 2002, 2003) to explain the large fraction of sdB stars in binaries. These scenarios involve strong mass transfer either through a common-envelope ejection or through stable Roche lobe overflow. A merger of two helium-core white dwarfs is another viable option.

The binary population synthesis models of Han et al. (2002) predict a mass distribution for sdB stars that peak near the canonical mass, but is much wider, ranging from $0.3 M_{\odot}$ to more than $0.7 M_{\odot}$. The lower limit is based on the fact that no core helium burning can be sustained for stars with masses less than $0.3 M_{\odot}$. The highest masses result from mergers. It might be premature to assume that the mass of an $\operatorname{sdB}$ star is half a solar mass, as has been done in previous studies. Therefore we drop this assumption and treat the mass of the $\mathrm{sdB}$ component as a free parameter.

Another drawback of all previous investigations was the lack of information on the inclination angle. We aim here for the first time at deriving constraints on the inclination by means of accurate measurements of the projected rotational velocity and the surface gravity from appropriate high-quality spectra using sophisticated model atmospheres. Because the rotation of the $\mathrm{sdB}$ star is tidally locked to the orbit, we can derive $\sin i$ as a function of the sdB mass from these two quantities. This approach, combined with a reanalysis of the published light curve, allows us to constrain the masses of both components and, therefore, to check whether or not KPD 1930+2752 qualifies as a SN Ia progenitor candidate through a DD merger.

Section 2 describes the newly obtained spectra, from which an improved radial velocity curve has been derived (Sect. 3). For the quantitative spectral analysis, existing grids of metal lineblanketed LTE models were used and a new (hybrid) approach applied that allows us to treat both departures from LTE, as well as metal line blanketing (Sect. 4). Combining the results described in Sect. 5 with the projected rotational velocity (Sect. 6), we constrained masses and inclination of the KPD 1930+2752 system (Sect. 7). Within the derived parameter space, synthetic light curves were modelled and fitted to the published light curve (Sect. 8) in order to constrain the parameters even further. Our conclusions are given in the final section.

\section{Observations and data reduction}

With the $10 \mathrm{~m}$ Keck I Telescope at the Mauna Kea Observatory two hundred high-resolution spectra were obtained by N. Przybilla in half a night in July 2004, using the High Resolution Echelle Spectrometer (HIRES; Vogt et al. 1994). The spectra covered a wavelength range of $4200 \AA-6600 \AA$ with a few small gaps at a resolution of $0.1 \AA$ and exposure times of $20 \mathrm{~s}$ each. The data were reduced using the ESO-MIDAS package. Bias and flatfield corrections were applied. The echelle orders were extracted and sky background subtracted. The wavelength calibration was done separately for every single order. All spectra were corrected to the heliocentric frame of reference. To perform model atmosphere fits, all spectra were radial-velocity corrected to rest wavelength and a coadded spectrum was generated separately for each echelle order. 


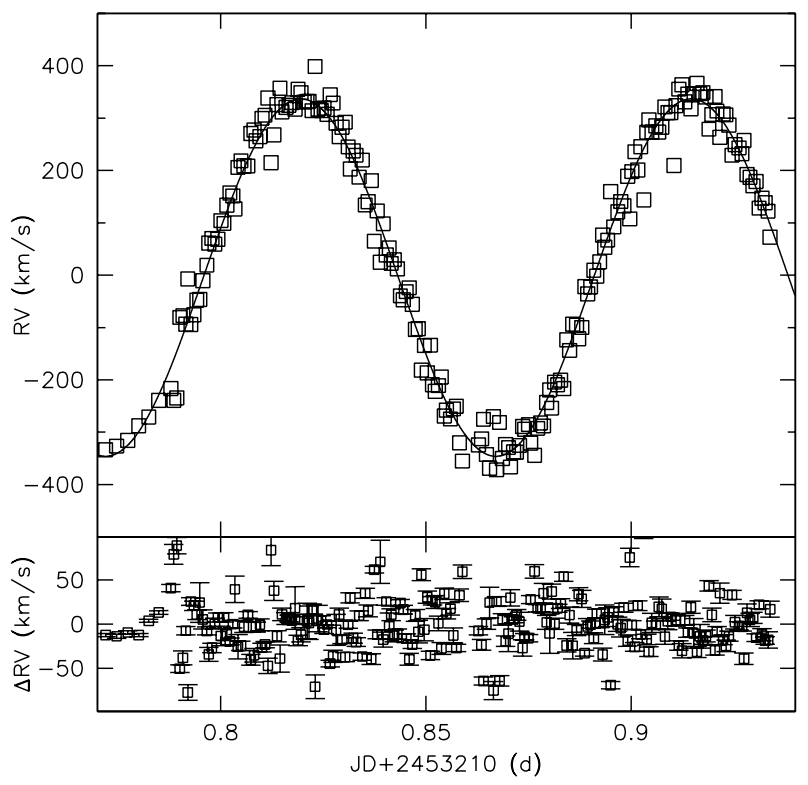

Fig. 1. Radial velocity curve. The data points are from the new HIRES spectra, and the solid line is the fitted sine curve to these points. The residuals in the lower part show no signs of eccentricity.

Two spectra were taken with the ESO Very Large Telescope UT2 (Kueyen) equipped with the UV-Visual Echelle Spectrograph (UVES) in 2001 (Dekker et al. 2000). The spectra cover a wavelength range of 3300-6650 ̊ with two small gaps at a resolution of $0.1 \AA$. The exposure time was $300 \mathrm{~s}$ each. The spectra were reduced with a pipeline developed by C. Karl using the ESO-MIDAS package and based on the implemented UVES reduction pipeline (Karl 2004). Additional observations were obtained with the $2.2 \mathrm{~m}$ Telescope at the Calar Alto Observatory in July 2004. The Calar Alto Faint Object Spectrograph (CAFOS) was used to obtain 150 spectra covering a wavelength range of $3600 \AA-6200 \AA$ with $5 \AA$ resolution and an exposure time of $180 \mathrm{~s}$ each. The data were reduced and coadded in analogy to the HIRES spectra.

\section{Radial velocities and spectroscopic orbit}

Because the $\mathrm{S} / \mathrm{N}$ ratio of the individual HIRES spectra was very low, only the $\mathrm{H} \alpha$ and $\mathrm{H} \beta$ lines could be used for determining the radial velocity by $\chi^{2}$ cross correlation with a model spectrum at rest wavelength. To improve the accuracy, the resulting radial velocity curve was combined with all available radial velocity data of KPD 1930+2752 (Maxted et al. 2000; Woolf et al. 2002; Orosz 2000 priv. comm.) covering a timespan of four years. A sine curve was fitted to these 2900 data points using an $\chi^{2}$ minimising method and the power spectrum was generated (FITRV and FITPOW routines by H. Drechsel; Napiwotzki et al. 2001). The sine curve fit is excellent for all different datasets and no period change could be detected over the whole timebase (Figs. 1 and 2). The orbital parameters were measured with unprecedented accuracy and are consistent with prior measurements by Maxted et al. (2000) and Woolf et al. (2002): $\gamma(\mathrm{H} \alpha)=$ $5 \pm 1 \mathrm{~km} \mathrm{~s}^{-1}, K=341 \pm 1 \mathrm{~km} \mathrm{~s}^{-1}, P=0.0950933 \pm 0.0000015 \mathrm{~d}$. The residuals do not show any signs of eccentricity.

No radial velocity variations due to pulsations could be found either. This result is consistent with the non-detection of RV variations by Woolf et al. (2002). The high orbital $\mathrm{RV}$-variation of the system enforces short exposure times in order to prevent orbital smearing effects. Considering that our spectra were obtained with the largest optical telescope in the world, it can be doubted that such a detection would be feasible for this object with present-day instrumentation.

\section{Atmospheric models and synthetic spectra}

Up to now spectra of sdB stars were analysed either from metal line-blanketed LTE model atmospheres or from NLTE model atmospheres neglecting metal line blanketing altogether. As pointed out by Heber et al. (2000) and Heber \& Edelmann (2004), systematic differences between these two approaches are present. Most importantly the gravity scale differs by about 0.06 dex. O'Toole \& Heber (2006) found very high abundances of heavy elements in pulsating sdB stars with similar temperature to KPD $1930+2752$ and showed that model atmospheres with supersolar metallicity $(10 \times$ solar $)$ reproduced the optical spectra much better than solar metallicity models did. As a result, we used their model grids for both solar and 10 times solar metallicity in the analysis of the spectra of KPD 1930+2752.

As the gravity is of utmost importance for our analysis, we also calculated new grids of models and synthetic spectra to account for NLTE effects and metal line blanketing simultaneously. Since the temperature/density structure of an sdB atmosphere is only slightly affected by NLTE effects (if at all), the LTE approximation is valid to this end. NLTE effects may become more important for the line formation of Balmer and helium lines. Therefore, we chose a "hybrid" approach by calculating the temperature/density stratification from metal line-blanketed LTE model atmospheres and then performing line formation calculations for hydrogen and helium, allowing for departures from LTE (Przybilla et al. 2006). We used both of the Kurucz codes, ATLAS9 and ATLAS12, to calculate metal line-blanketed model atmospheres (Kurucz 1993, 1996). The coupled statistical equilibrium and radiative transfer equations were solved and spectrum synthesis with refined line-broadening theories was performed using DETAIL and SURFACE (Giddings 1981; Butler \& Giddings 1985). Both codes have undergone major revisions and improvements over the past few years. State-ofthe-art NLTE model atoms for hydrogen and helium are utilised (Przybilla \& Butler 2004; Przybilla 2005).

The Stark broadening of hydrogen lines is important for determining temperature and gravity. Up to now all spectral analyses of sdB stars have used synthetic Balmer-line spectra based on the unified theory of Vidal et al. (1973, VCS) with the improvements of Lemke (1997). Since new broadening tables for Balmer lines have become available (Stehlé \& Hutcheon 1999, $\mathrm{SH}$ ), we also calculated synthetic spectra from these tables to investigate systematic effects on the synthetic spectra.

\section{Atmospheric parameter determination}

The CAFOS spectra were preferred for the parameter determination because they have excellent $\mathrm{S} / \mathrm{N}$ and the run of the continuum is smooth. The continuum of the Keck HIRES spectrum suffers from a non-optimal rectification of the continuum, which rendered the analysis of the Balmer line wings difficult or unreliable. Therefore we restrained ourselves from determining atmospheric parameters from the Keck HIRES spectra.

The observed spectra were analysed with FITPROF by means of an $\chi^{2}$ fit (Napiwotzki 1999). Rotational broadening was accounted for by choosing an appropriate value for the projected rotational velocity (see Sect. 6). 


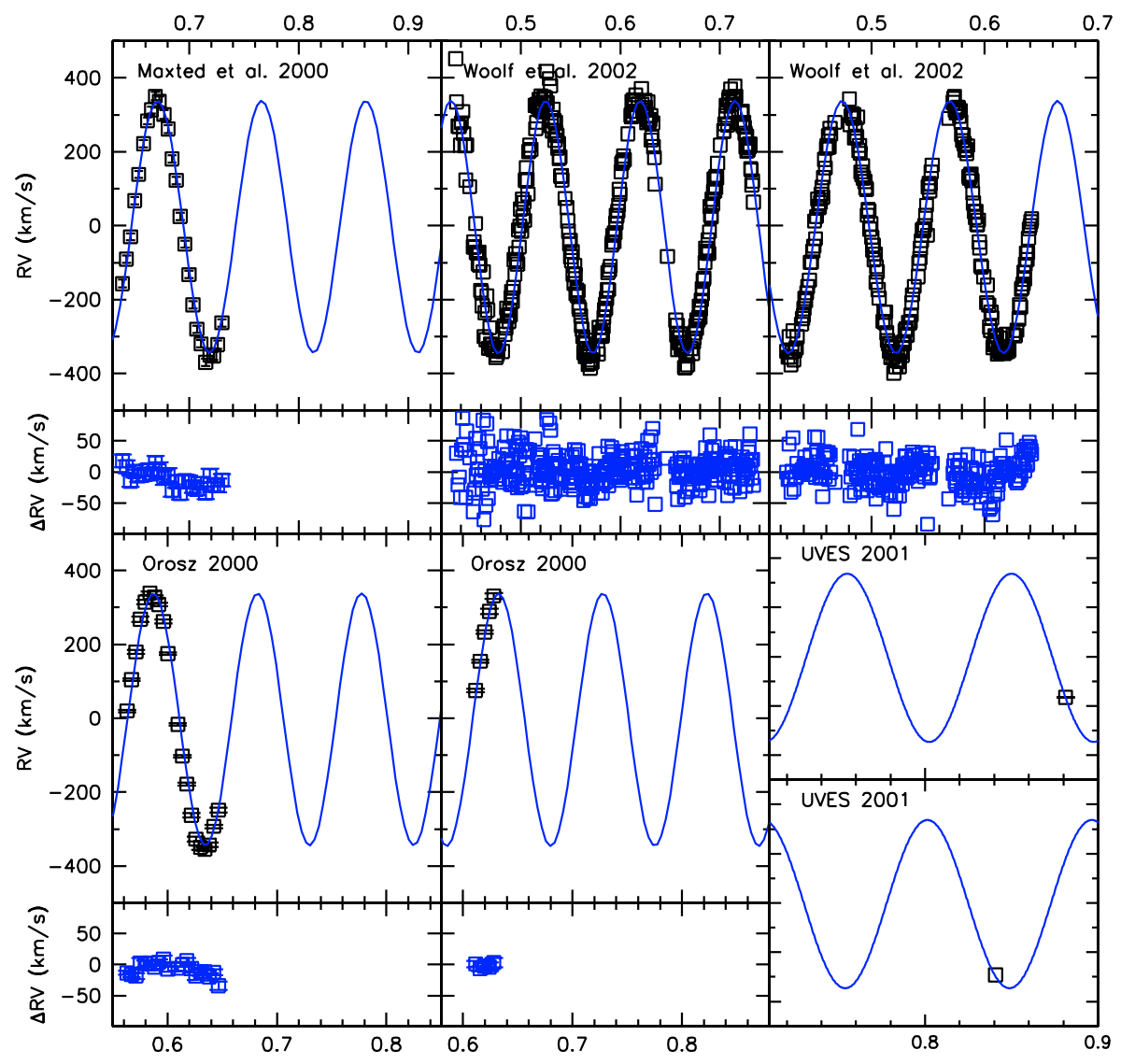

Fig. 2. Radial velocity curves for the years 2000 and 2001. Times are given in days. The corresponding HJD has to be added. Data are from Maxted et al. (2000: HJD = 2451651), Woolf et al. (2002: HJD = 2451716/2451717), Orosz (priv comm.: $\mathrm{HJD}=2451769 / 2451770)$, and from UVES spectra $(\mathrm{HJD}=2452033 / 2452065)$. The solid line is the fitted sine curve to all data points over a timespan of four years. The residuals in the lower parts show no signs of eccentricity. The error bars in the curve of Woolf et al. have been skipped, because they are very large due to the low $\mathrm{S} / \mathrm{N}$ ratio of the spectra.
Motivated by the discovery of strong $\log g$ variations in the pulsating subdwarf star PG 1325+101 (Telting \& Østensen 2004), this possibility was also taken into account. To search for changes of the stellar parameters due to pulsations or due to the ellipsoidal deformation of the subdwarf star, three to ten CAFOS spectra were coadded. The atmospheric parameters for each phase were determined. No changes could be detected. Then we combined all CAFOS spectra to form a very high $\mathrm{S} / \mathrm{N}$ spectrum and performed a fit. The formal statistical errors were small, i.e. $\Delta T_{\mathrm{eff}} \approx 100 \mathrm{~K}, \Delta \log g \approx 0.005 \mathrm{dex}, \Delta \log \frac{n(\mathrm{He})}{n(\mathrm{H})} \approx 0.005 \mathrm{dex}$. However, systematic errors due to inaccuracies in the model atmospheres and synthetic spectra may be much larger. To investigate this we used the different grids of models described in Sect. 4 to determine the atmospheric parameters.

We started the analysis by using the metal line-blanketed LTE model atmosphere grids of O'Toole \& Heber (2006). A simultaneous fit of the hydrogen and some helium lines (He I $4026 \AA$, He II $5412 \AA$ and in particular He II $4686 \AA$ ) was not possible with solar metallicity models (Fit A in Table 1). This so-called helium problem already occurred during other analyses of pulsating subdwarfs (Heber et al. 2000; Edelmann 2003). The analysis of HST-UV spectra of three sdB stars with similar $T_{\text {eff }}$ as KPD 1930+2752 revealed supersolar abundances of the iron group elements (O'Toole \& Heber 2006). Those stars also displayed the optical He ionisation problem. With more appropriate metal-rich models (10 times solar metallicity), the problem could be remedied because of a modified atmospheric temperature structure due to significantly increased line blanketing (see also Heber et al. 2006). The abundances of the iron group elements could not be determined for KPD 1930+2752 due to its high rotational velocity, which causes a strong broadening of all metal lines. Because of the similarity of its atmospheric
Table 1. Atmospheric parameters of KPD $1930+2752$ derived from model atmosphere fits, where A12 = Kurucz ATLAS12 code, VCS = Vidal, Cooper \& Smith (1973), SH = Stehlé \& Hutcheon (1999), lf = line formation.

\begin{tabular}{lllccc}
\hline \hline & model & {$[m / H]$} & $T_{\text {eff }}$ & $\log g$ & $\log \frac{n(\mathrm{He})}{n(\mathrm{H})}$ \\
\hline A & LTE VCS & 0 & $35720 \mathrm{~K}$ & 5.67 & -1.56 \\
B & LTE VCS & +1 & $35183 \mathrm{~K}$ & 5.55 & -1.47 \\
C & NLTE (lf) VCS & +1 & $35353 \mathrm{~K}$ & 5.61 & -1.50 \\
D & NLTE (lf) SH & +1 & $35212 \mathrm{~K}$ & 5.67 & -1.51 \\
E & NLTE (lf) SH A12 & +1 & $35712 \mathrm{~K}$ & 5.67 & -1.58 \\
\hline & adopted & & $35200 \mathrm{~K}$ & 5.61 & -1.50 \\
& & & \pm 500 & \pm 0.06 & \pm 0.02 \\
\hline
\end{tabular}

parameters to those of the stars studied by O'Toole and Heber (2006), we adopted high-metallicity models as well and, the fit indeed improved (Fit B in Table 1, Figs. 4 and 3).

In a second step we used the new grid of those hybrid models that account for both metal line blanketing and departures from LTE using ATLAS9. We chose supersolar metallicity because of the experience with the LTE analysis described above. The quality of this fit (labelled $\mathrm{C}$ in Table 1) is comparable to that from the LTE analysis.

Then we checked the influence of Balmer line broadening by using synthetic spectra calculated from ATLAS9 models, 10 times solar metallicity with the hybrid approach and $\mathrm{SH}$ broadening tables instead of VCS tables (Fit D in Table 1). Finally ATLAS9 was replaced by ATLAS12 and the analysis repeated (Fit E in Table 1) using the same approach as in the previous step.

The resulting parameters from the different grids are summarised in Table 1. As can be seen, systematic effects on the 


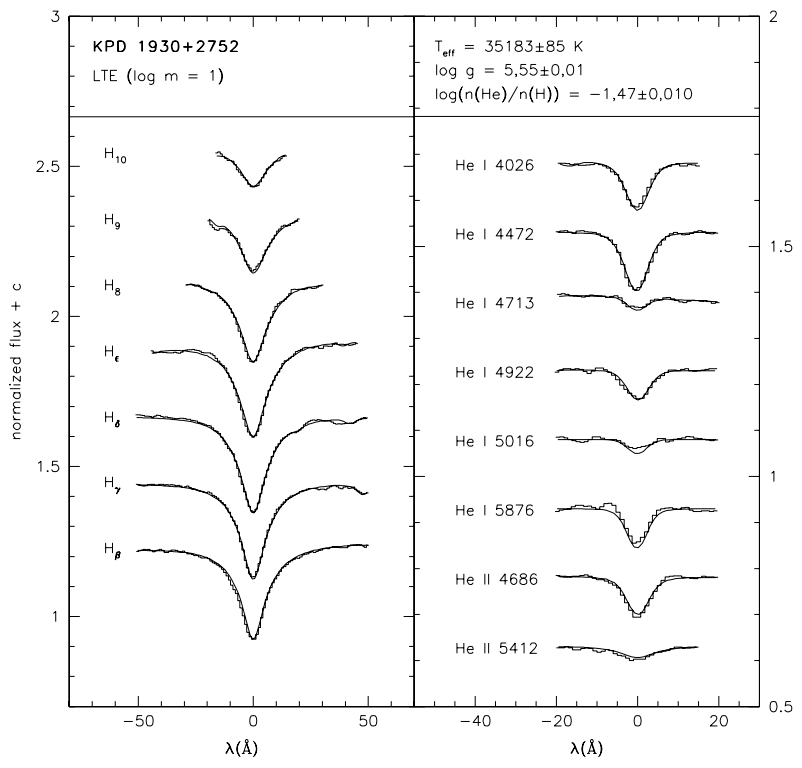

Fig. 3. Best fit with an LTE model of ten times solar metallicity (model B of Table 1).

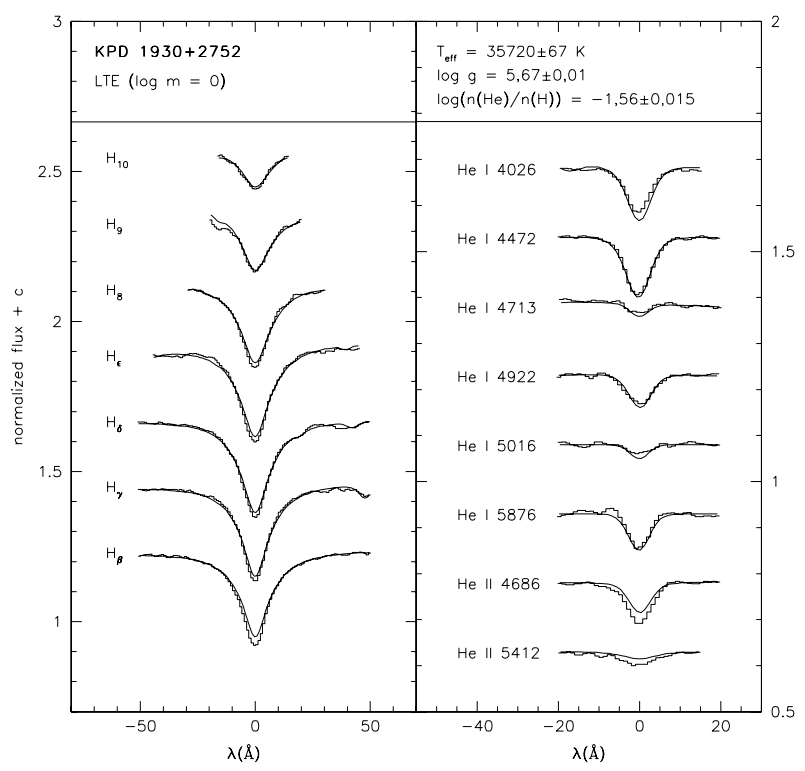

Fig. 4. Fit with an LTE model grid of solar metallicity (model A of Table 1).

effective temperature are small, as models A to E differ by $530 \mathrm{~K}$ or less. The gravities, however, differ by as much as $0.12 \mathrm{dex}$. The "hybrid" models yield a gravity that is 0.06 dex higher than do the LTE models for supersolar metal content. The replacement of the VCS tables by SH tables systematically increases gravity by another 0.06 dex. It is possible that part of the shift is explained by differences in table organisation and interpolation (Lemke 1997). Note in passing that the solar metallicity LTE model (VCS broadening tables), i.e. the one with the least sophistication, yields almost the same $T_{\text {eff }}$ and gravity as the most sophisticated model grid (supersolar ATLAS12 plus NLTE line formation plus SH line broadening). However, the fit of the observed spectrum from the latter grid is superior. It is impossible to judge which of the models in Table 1 is preferable. The quality of fit lends support to synthetic spectra calculated from supersolar metallicity models. We therefore dismissed fit A (calculated for solar metallicity) and adopted the mean values of the other fits: $T_{\text {eff }}=35200 \pm 500 \mathrm{~K}$ and $\log g=5.61 \pm 0.06$ dex. For the sake of completeness we mention that the helium abundance differs by 0.11 dex.

It is important to note that the effective temperature is significantly higher (about $2000 \mathrm{~K}$ ) than previously derived in other investigations (Billères et al. 2000), irrespective of the choice of model atmosphere. Thus, KPD $1930+2752$ is situated at the hot edge of the instability strip in the $T_{\text {eff }}-\log g$ diagram (Charpinet et al. 1996).

\section{Projected rotational velocity}

The primary aim of the high-resolution time-series spectroscopy was to measure the projected rotational velocity of KPD $1930+2752$ as accurately as possible. For this purpose, a high S/N spectrum was constructed from the 200 HIRES spectra. Beforehand, the individual spectra were shifted to rest wavelength. The median was calculated in order to filter cosmics.

The projected rotational velocity was measured by convolving a synthetic spectrum calculated from the best-fit model atmosphere with a rotational broadening ellipse for appropriate $v_{\text {rot }} \sin i$. The FITSB2 routine of R. Napiwotzki was used, which fits the projected rotational velocity and performs an error calculation based on a bootstrapping algorithm (Napiwotzki et al. 2004). The lines $\mathrm{H} \alpha, \mathrm{H} \beta, \mathrm{H} \gamma, \mathrm{He}$ II $4686 \AA$, He I $4472 \AA$, He I $4922 \AA$, and He I $5016 \AA$ were used simultaneously for this measurement. The result is $v_{\text {rot }} \sin i=93.8 \pm 2.2 \mathrm{~km} \mathrm{~s}^{-1}$.

Fortunately, one of the two UVES spectra was taken near the maximum of the radial velocity curve (see Fig. 2 lower right corner). That means that, despite the relatively long exposure time, orbital smearing is negligible and the spectrum can be used for the $v_{\text {rot }} \sin i$-measurement, too. The rotational velocity was obtained as described above from the lines $\mathrm{H} \alpha-$ H8, He II $4686 \AA$, He I $4026 \AA$, He I $4472 \AA$, He I $4922 \AA$, and He I $5016 \AA$. The measured $v_{\text {rot }} \sin i=90.7 \pm 2.1 \mathrm{~km} \mathrm{~s}^{-1}$ is consistent with the HIRES result. To get a smaller error margin, we calculated the average of the two independent measurements $v_{\text {rot }} \sin i=92.3 \pm 1.5 \mathrm{~km} \mathrm{~s}^{-1}$ (see Fig. 5).

\section{Mass and inclination}

KPD $1930+2752$ is obviously affected by the gravitional forces of the companion, demonstrated by its ellipsoidal deformation. Since the period of the photometric variations is exactly half the period of the radial orbit and the two components of the binary are close together, the rotation of the $\mathrm{sdB}$ star is very likely tidally locked to the orbit. Having determined the gravity and projected rotational velocity, we have three equations at hand that constrain the system, with the sdB mass $M_{\mathrm{sdB}}$ the only free parameter. Besides the mass function

$f\left(M_{\mathrm{sdB}}, M_{\mathrm{comp}}\right)=\frac{M_{\mathrm{comp}}^{3}(\sin i)^{3}}{\left(M_{\mathrm{sdB}}+M_{\mathrm{comp}}\right)^{2}}=\frac{K_{\mathrm{sdB}}^{3} P}{2 \pi G}$,

these are

$\sin i=\frac{v_{\text {rotsini }} P}{2 \pi R}$

$R=\sqrt{\frac{M_{\mathrm{sdB}} G}{g}}$

With $\log g$ obtained from the model atmosphere analysis, the radius of the star $R$ was calculated using the standard mass-radius 


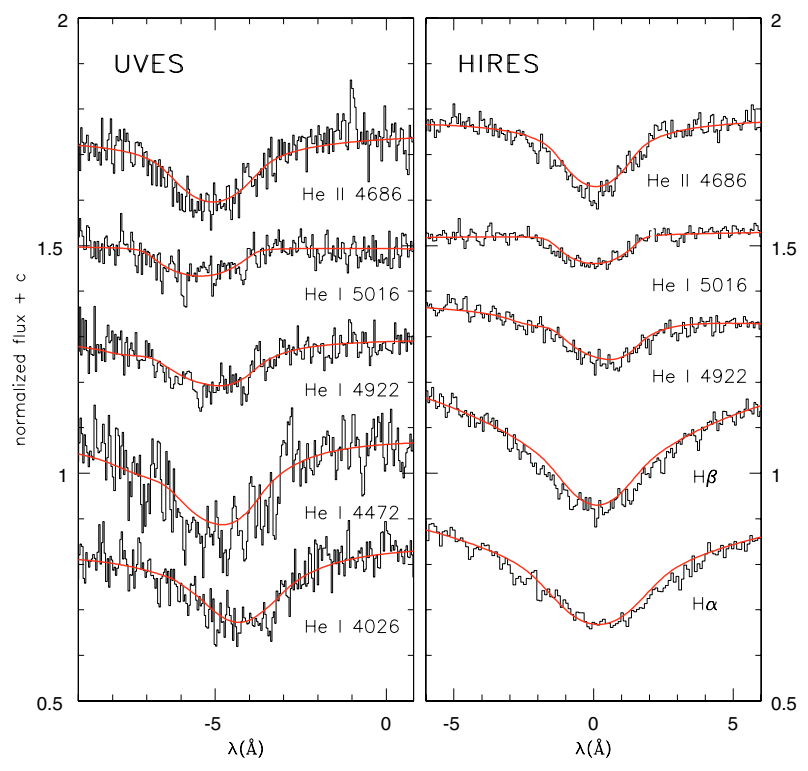

Fig. 5. Best fits of $v_{\text {rot }} \sin i$ to some lines of the HIRES and UVES spectra. The atmospheric parameters were fixed to the values derived from the CAFOS spectra. LTE models with NLTE line formation and ten times solar metallicity were used (model grid C, Table 1 ).

relation (Eq. (3)). Together with the orbital period of the system $P$ and the projected rotational velocity $v_{\text {rotsini }}=v_{\text {rot }} \sin i$, the inclination of the system $\sin i$ was derived for different values of the sdB mass. Because the rotation is tidally locked to the orbit, the rotational period of the sdB equals the orbital period of the system, so the absolute value of the rotational velocity could be calculated. The inclination of the system was then derived from the measured projected rotational velocity (Eq. (2)). With the sdB mass as free parameter, the measured radial velocity semiamplitude $K_{\mathrm{sdB}}$ and orbital period $P$ the mass function was solved numerically (Eq. (1)) to derive the mass of the companion $M_{\text {comp }}$ and calculate the total mass of the binary. That $\sin i$ cannot exceed unity gave a lower limit for the mass of the $\operatorname{sdB} M_{\mathrm{sdB}} \geq 0.45 M_{\odot}$ (see Fig. 6). The errors were calculated with Gaussian error propagation. The error budget is dominated by the error in $\log g$, which has to be estimated from the model atmosphere fit. Period $P$, radial velocity semiamplitude $K_{\mathrm{sdB}}$, and projected rotational velocity $v_{\text {rot }} \sin i$ are actually more precise than required for our measurements. This means that the accuracy of the results cannot be improved before better model grids become available. The higher value of $\log g$, which was obtained by using new line broadening tables, indicates a shift in the mass minimum and therefore of the total binary mass towards higher values. As can be seen in Fig. 7, the total mass of the system exceeds the Chandrasekhar limit for almost all assumptions of $M_{\mathrm{sdB}}$. If the companion is a white dwarf, its single mass has to be lower than the Chandrasekhar limit. This implies an upper limit $M_{\mathrm{sdB}} \leq 0.64 M_{\odot}$ and a possible total mass range of $M_{\mathrm{sdB}+\mathrm{WD}}=1.33 \pm 0.08-2.04 \pm 0.14 M_{\odot}$ (Fig. 7). A more massive invisible companion, such as a neutron star or a black hole, cannot be ruled out completely yet. In this case the subdwarf mass would exceed the possible mass range for this kind of star (Han et al. 2002, 2003). The inclination angle of the system (Fig. 6) is close to $90^{\circ}$. KPD $1930+2752$ could be an eclipsing binary (cf. the very similar binary KPD $0422+5421$, Orosz \& Wade 1999). The detection of eclipses would rule out any object smaller than a white dwarf. If eclipses were detected, the sdB mass would be constrained to $0.45 M_{\odot}$ to $0.54 M_{\odot}$ (see

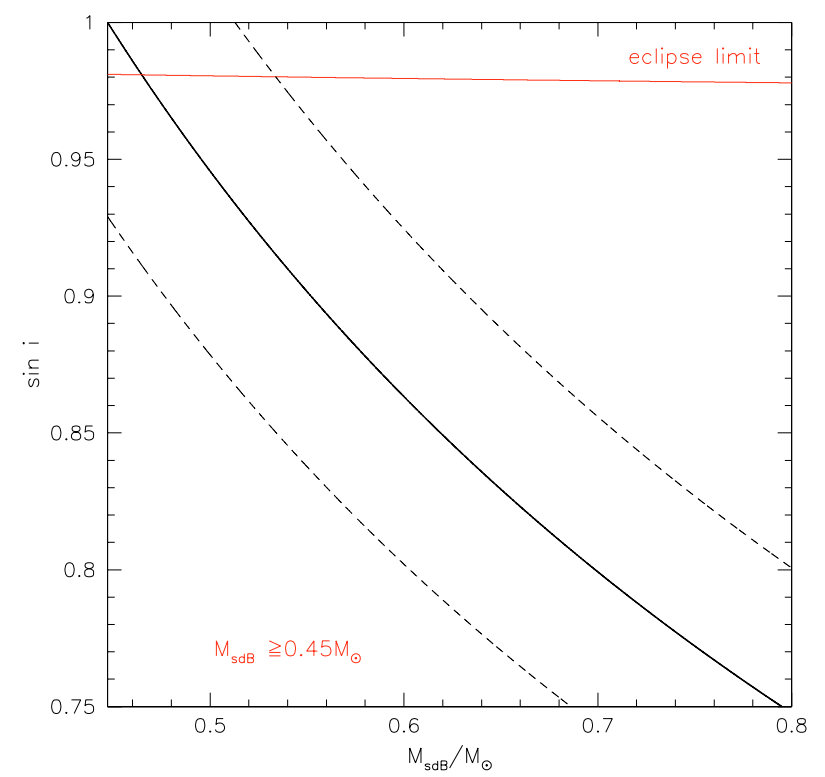

Fig. 6. Inclination versus $\mathrm{sdB}$ mass. The dashed lines correspond to $\pm 1 \sigma$ error. The upper curve indicates the minimum inclination for an eclipse. Since $\sin i$ cannot exceed unity, a minimum mass for the sdB follows.

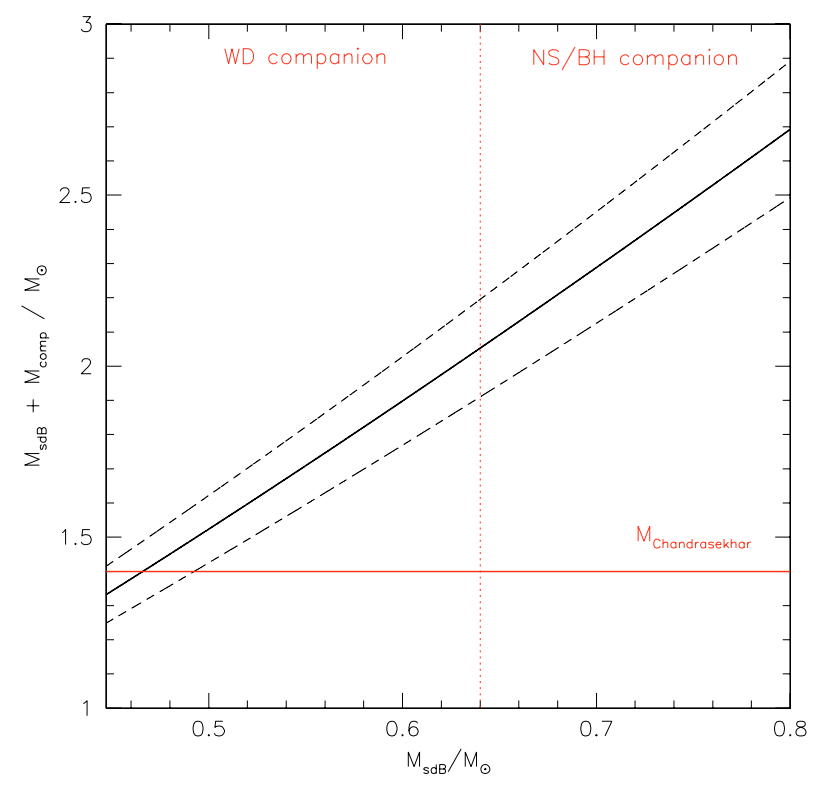

Fig. 7. Total mass of the binary system as a function of the sdB mass with associated error margins (dashed lines) for the total mass. The horizontal line marks the Chandrasekhar limit. The dotted vertical line marks the point where the companion mass equals this limit. For higher $\mathrm{sdB}$ masses, the companion cannot be a white dwarf, but has to be a heavier object such as a neutron star (NS) or a black hole (BH).

Fig. 6). On the other hand, if eclipses could be ruled out from high quality light curves, the total mass of the system must be higher than $1.55 M_{\odot}$, well above the Chandrasekhar limit.

\section{Constraints by photometry}

The light curve obtained by Billères et al. (2000) shows ellipsoidal variations. We used this information to further constrain the parameters of the KPD 1930+2752 system. We employed the light curve synthesis and solution code MORO based on the model by Wilson \& Devinney (1971). The details of the 


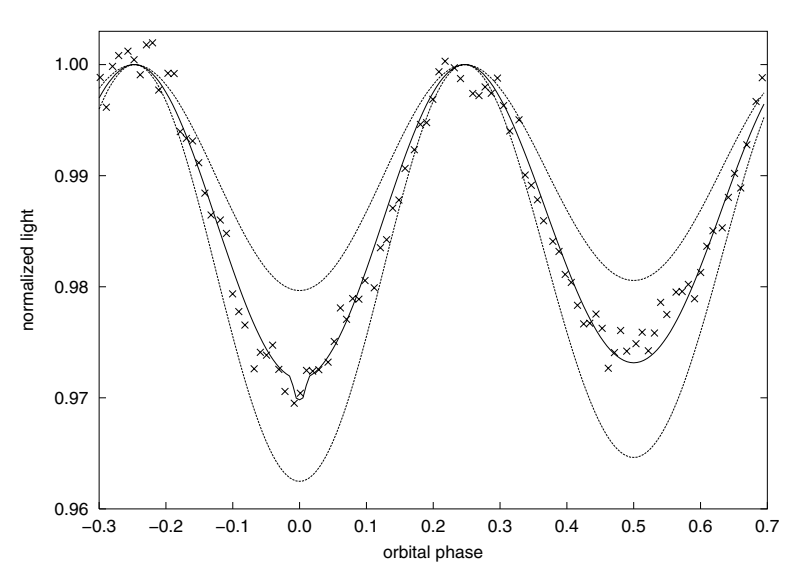

Fig. 8. Light curve data taken from Billères et al. (2000) shown together with examples of synthetic light curves. A good fit $\left(M_{\mathrm{sdB}} \approx 0.47 M_{\odot}\right.$; solid line) is compared to light curves for $M_{\mathrm{sdB}} \approx 0.45 M_{\odot}$ and $M_{\mathrm{sdB}} \approx$ $0.55 M_{\odot}$. For these two cases the other parameters have been calculated according to Eqs. (1) to (3).

Bamberg implementation are given by Drechsel et al. (1995). The software uses a modified Roche model for light curve synthesis. It is capable of realistically simulating the distortions of the stars induced by the presence of a companion.

For comparison of the observations with the synthetic light curves of MORO, it was necessary to prepare the data accordingly. Since the program does not include the effect of Doppler boosting arising from the high orbital velocity, this effect had to be corrected for. It increases the total flux by a factor of $(1-v(t) / c)^{3}$, where $v$ is the radial velocity at time $t$ and $c$ the speed of light. The boosting is counteracted by the Doppler shift, which reduces the effect by a factor of $(1-v(t) / c)^{2}$ (see Maxted et al. 2000). A resulting factor of $(1-v(t) / c)$ was applied to the total flux. Furthermore, the light curve shows a dip during the maximum at orbital phase 0.25 . This effect is currently unexplained and cannot be modelled by MORO, therefore the corresponding data points have been left out for the light curve analysis. Because KPD $1930+2752$ is a pulsating subdwarf with a very rich spectrum of modes, the short period pulsations have been filtered out by Billères et al. (2000). As this procedure is not trivial at all, hidden pulsations may limit the accuracy of the results. On the other hand, the amplitude of the ellipsoidal variations is very sensitive to the system parameters, as can be seen in Fig. 8.

In a first trial, light curves with different component masses and orbital inclinations were synthesised. The corresponding changes in the light curve shapes were significant enough to encourage further investigation. A coarse grid of light curves was then synthesised, which covered the parameter space between $M_{\mathrm{sdB}}=0.45 M_{\odot}$ and $M_{\mathrm{sdB}}=1.0 M_{\odot}$. For $\sin i$ and $R_{\mathrm{sdB}}$ a range of values was calculated for each $M_{\mathrm{sdB}}$ according to Eqs. (1) to (3). These ranges covered the parameter space between the respective error limits derived from spectroscopy. Other relevant parameters for light curve synthesis, like orbital separation $a$ and the companion mass, were derived from Kepler's laws of orbital motion. The radius of the companion was calculated from the mass-radius-relation for white dwarfs, or set to an infinitesimal value for neutron star masses (in that case ruling out eclipse effects in the light curves). An $\chi^{2}$ value was calculated for each grid point with respect to the observed light curve. A coarse analysis revealed the marked tendency of good fits to cluster in the sin $i$ regions of marginal eclipse. According to Eqs. (2) and (3)

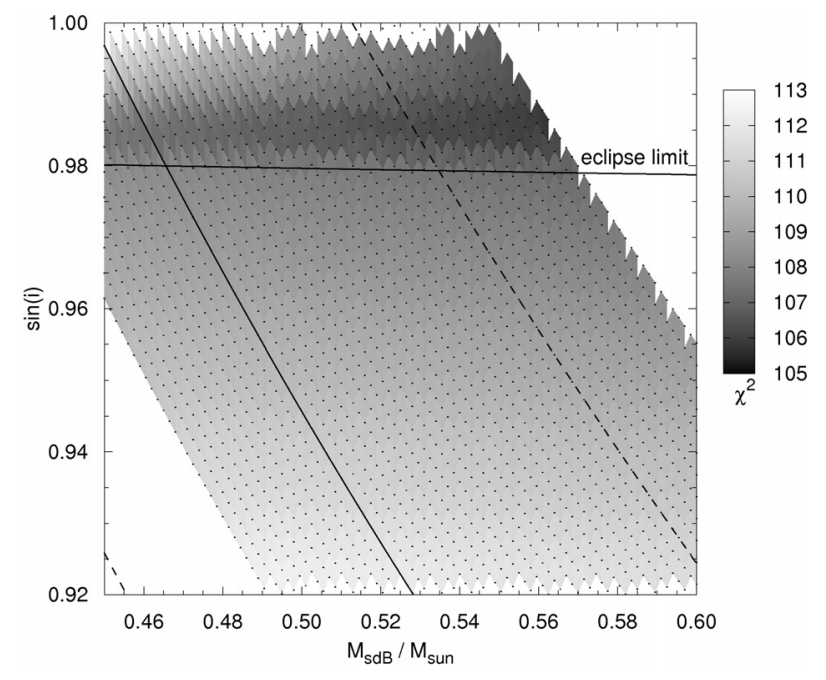

Fig. 9. Detail of Fig. 6 with light curve data superimposed. The full drawn line is the relation derived from spectroscopy. The dashed lines mark the errors in $\sin i$. Each dot denotes the location of a parameter set for which a light curve has been calculated. The background shading corresponds to the quality of the fit - darker shading implying better $\chi^{2}$. Note that good solutions cluster slightly above the eclipse limit, indicating a high orbital inclination near $80^{\circ}$.

this behaviour strongly favored $\mathrm{sdB}$ masses close to the canonical value (Fig. 10). Lower values of $\sin i$ and higher sdB masses could be ruled out quickly, since they led the to quick deterioration of fit quality.

In order to probe the relevant portion of the parameter space in more detail, a very fine grid of synthetic light curves was constructed, containing more than 76000 parameter combinations. It covered only that area of parameter space where promising solutions had been identified previously. The range of sdB masses extended from $0.45 M_{\odot}$ to $0.60 M_{\odot}$. Indeed, a significant overlap between the spectroscopically determined parameter range and a set of very good light curve fits could be found (see Fig. 9). Most notable is the coincidence of the best fits with the region of eclipse $\left(i \approx 80^{\circ}\right)$. Fit quality deteriorates significantly for lower inclinations. Probably this behaviour stems from the light curve minimum at orbital phase 0 , which shows signs of an eclipse effect and is therefore fit marginally better by synthetic light curves displaying the same effect. On the other hand, this marginal dip could also be a remnant from removing the intrinsic stellar pulsations. A better light curve is needed to decide whether the binary is eclipsing or not.

As noted previously, the light curve fits, favouring an eclipse and therefore a high inclination, constrain the possible system mass considerably. However, the only absolute parameter that can be determined from the light curves alone is the orbital inclination, as the results of light curve analyses are well known to be degenerate with respect to the system scale. Indeed, the $\mathrm{sdB}$ mass is poorly constrained by the light curve fit (see Fig. 9), as are the orbital separation and the radii of the stars. However, if we combine the results of the photometric analysis with the mass-inclination relation derived from spectroscopy (Fig. 6), the sdB mass is constrained to a very narrow range of $M_{\mathrm{sdB}}=0.45-0.52 M_{\odot}$, corresponding to a total mass of 1.36-1.48 $M_{\odot}$ (see Fig. 10). On the other hand, sdB masses higher than $M_{\mathrm{sdB}} \approx 0.52 M_{\odot}$ can be discarded since, in that part of the parameter space, the spectroscopic mass relation does not overlap with good light curve fits within the error margins. A neutron star companion can therefore be ruled out. 


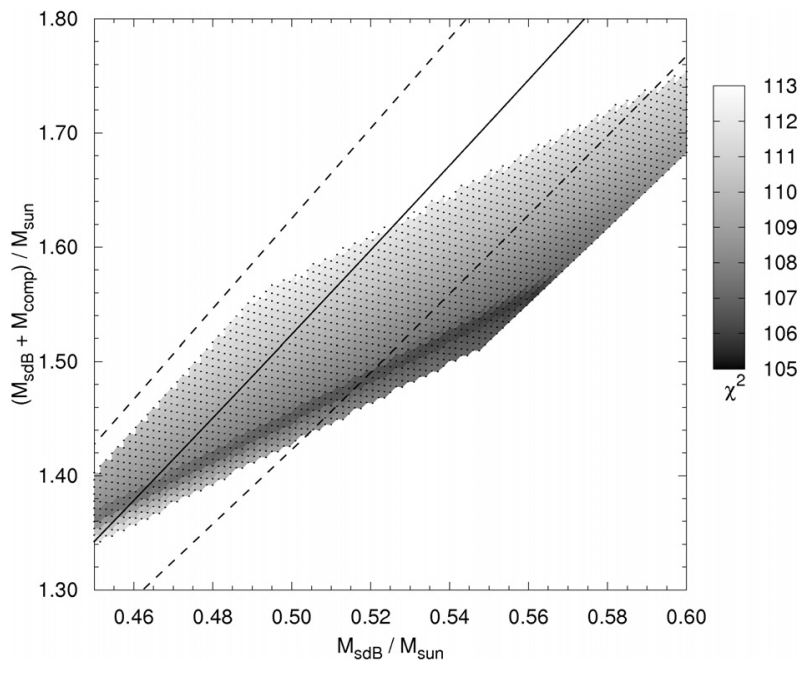

Fig. 10. Detail of Fig. 7 with light curve data superimposed. The full drawn line is the relation derived from spectroscopy. The dashed lines mark the errors in total mass. Each dot denotes the location of a parameter set for which a light curve has been calculated. The background shading corresponds to the quality of the fit - darker shading implying better $\chi^{2}$.

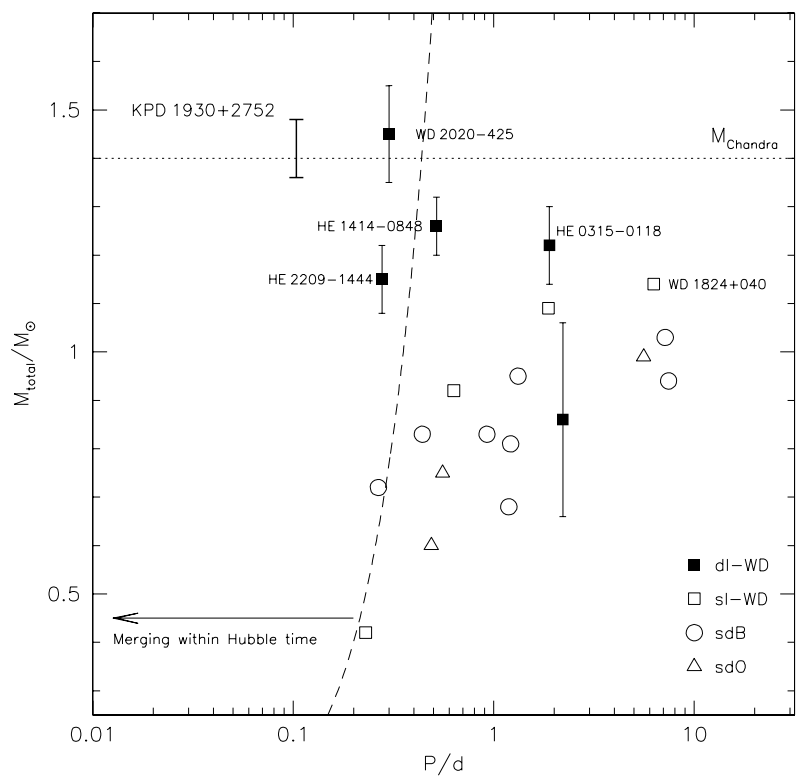

Fig. 11. Total mass plotted against logarithmic period of DD systems from the SPY survey. The filled rectangles mark double lined WDs, for which the absolute mass can be derived. The open symbols mark singlelined WDs, sdBs, and sdOs. Only lower mass limits can be derived for these binaries by adopting $\sin i=1$ (Napiwotzki et al. 2005; Karl 2004; Napiwotzki et al. 2002; Karl et al. 2003).

\section{Conclusion}

The combination of an extensive set of 350 high- and lowresolution spectra, 2700 radial velocity data points from prior observations, and a reanalysis of the published light curve made it possible to determine the parameters of KPD 1930+2752 with unprecedented accuracy. The companion is a heavy white dwarf. KPD $1930+2752$ is the first subdwarf whose mass could be constrained in this way. With $0.45-0.52 M_{\odot}$, it is remarkably close to the canonical mass of $0.5 M_{\odot}$. It is worthwhile noting that masses of other sdBV derived by asteroseismology are also close to the canonical value (Brassard et al. 2001;
Charpinet et al. 2005a,b). Furthermore, Orosz \& Wade (1999) determine an sdB mass of $0.51 \pm 0.05 M_{\odot}$ for the eclipsing sdB+WD binary KPD $0422+5421$.

The total mass and the merging time of the binary indicate that it is an excellent DD candidate for an SN Ia progenitor. KPD $1930+2752$ is one of only two DD systems that fulfill these requirements (see Fig. 11; Napiwotzki et al. 2005). While it fits into the DD scenario, KPD 1930+2752 might also evolve into an SN Ia as an SD binary system. Since the time for merging due to gravitational wave radiation $t_{\text {merger }} \approx 2 \times 10^{8} \mathrm{yr}$ is of the same order as the EHB life time $t_{\mathrm{EHB}} \approx 1 \times 10^{8} \mathrm{yr}$, Roche lobe overflow could occur well before the sdB becomes a white dwarf due to the shrinkage of the orbit. Recent calculations by Han and Podsiadlowski (priv. comm.) indicate that the mass transfer would be stable.

Follow-up observations should be undertaken to measure an improved light curve and search for clear signs of eclipses. A promising alternative option is provided for asteroseismology. KPD $1930+2752$ is the first pulsating subdwarf with a mass estimate from orbital analysis. It could be used to calibrate asteroseismological models (see e.g. Charpinet et al. 2005b). This is a very difficult task because the frequency spectrum is very complex and modelling is rendered difficult by the ellipsoidal deformation and the rapid rotation of KPD 1930+2752.

Acknowledgements. We would like to thank C. S. Jeffery, P. F. L. Maxted, and J. Orosz for providing us with their data. We are also grateful to Z. Han and P. Podsiadlowski for their comments and interpretations of our results. SG was partly supported by the Deutsches Zentrum für Luft- und Raumfahrt (DLR) through grant no. 50-OR-0202. The authors wish to recognise and acknowledge the very significant cultural role and reverence that the summit of Mauna Kea has always had within the indigenous Hawaiian community. We are most fortunate to have the opportunity to conduct observations from this mountain.

\section{References}

Bennett, C. L., Halpern, M., Hinshaw, G., et al. 2003, ApJS, 148, 1 Billères, M., Fontaine, G., Brassard, P., et al. 2000, ApJ, 530, 441 Brassard, P., Fontaine, G., Billères, M., et al. 2001, ApJ, 563, 1013 Butler, K., \& Giddings, J. R. 1985, in Newsletter on Analysis of Astronomical Spectra, No. 9 (London: Univ. London)

Charpinet, S., Fontaine, G., Brassard, P., \& Dorman, B. 1996, ApJ, 471, L103

Charpinet, S., Fontaine, G., Brassard, P., et al. 2005a, A\&A, 437, 575

Charpinet, S., Fontaine, G., Brassard, P., et al. 2005b, A\&A, 443, 251

Dekker, H., D’Odorico, S., Kaufer, A., et al. 2000, Proc. SPIE, 4008, 534

Downes, R. A. 1986, ApJS, 61, 569

Drechsel, H., Haas, S., Lorenz, R., \& Gayler, S. 1995, A\&A, 294, 723

Dreizler, S., Heber, U., Werner, K., et al. 1990, A\&A, 235, 234

Edelmann, H. 2003, Ph.D. Thesis, Univ. Erlangen-Nuremberg

Ergma, E., Fedorova, A. V., \& Yungelson, L. R. 2001, A\&A, 376, L9

Giddings J. R. 1981, Ph.D. Thesis, Univ. London

Hamada, T., \& Salpeter, E. E. 1961, ApJ, 134, 638

Han, Z., Podsiadlowski, P., Maxted, P. F. L., Marsh, T. R., \& Ivanova, N. 2002, MNRAS, 336, 449

Han, Z., Podsiadlowski, P., Maxted, P. F. L., \& Marsh, T. R. 2003, MNRAS, 341, 669

Heber, U. 1986, A\&A, 155, 33

Heber, U., \& Edelmann, H. 2004, Ap\&SS, 291, 341

Heber, U., Reid, I. N., \& Werner, K. 2000, A\&A, 363, 198

Heber, U., Hirsch, H., Ströer, A., et al. 2006, in Proc. of the Second Meeting on Hot Subdwarf Stars, Baltic Astron., 15, 91

Iben, I. Jr., \& Tutukov, A. V. 1984, ApJS, 54, 335

Karl, C. 2004, Ph.D. Thesis, Univ. Erlangen-Nuremberg

Karl, C., Napiwotzki, R., Nelemans, G., et al. 2003, A\&A, 410, 663

Kilkenny, D., Koen, C., O’Donoghue, D., \& Stobie, R. S. 1997, MNRAS, 285, 640

Koen, C., Kilkenny, D., O’Donoghue, D., Van Wyk, F., \& Stobie, R. S. 1997, MNRAS, 285, 645

Kurucz, R. L. 1993, Kurucz CD-ROM No. 13 (Cambridge, Mass.: Smithsonian Astrophysical Observatory)

Kurucz, R. L. 1996, in Model Atmospheres and Spectrum Synthesis, ed. S. J. Adelman, F. Kupka, \& W. W. Weiss (San Francisco: ASP), 160 
Leibundgut, B. 2001, ARA\&A, 39, 67

Lemke, M. 1997, A\&AS, 122, 285

Livio, M. 2000, in Type Ia Supernovae: Theory and Cosmology (Cambridge Univ. Press), ed. J. C. Niemeyer, \& J. W. Truran, 33

Maxted, P. F. L., Marsh, T. R., \& North, R. C. 2000, MNRAS, 317, L41

Napiwotzki, R. 1999, A\&A, 350, 101

Napiwotzki, R., Edelmann H., Heber, U., et al. 2001, A\&A, 378, L17

Napiwotzki, R., Koester, D., Nelemans, G., et al. 2002, A\&A, 386, 957

Napiwotzki, R., Christlieb, N., Drechsel, H., et al. 2003, ESO Msngr, 112, 25

Napiwotzki, R., Yungelson, L., Nelemans, G., et al. 2004, in Spectroscopically and Spatially Resolving the Components of the Close Binary Stars, Proc. of the Workshop held 20-24 October 2003 in Dubrovnik, Croatia, ed. R. W. Hilditch, H. Hensberge, \& K. Pavlovski, ASP Conf. Ser., 318, 402

Napiwotzki, R., Karl, C. A., Nelemans, G., et al. 2005, in Proc. of the 14th European Workshop on White Dwarfs, ed. D. Koester, \& S. Moehler, ASP Conf. Ser., 334, 375

Orosz, J. A., \& Wade, R. A. 1999, MNRAS, 310, 773

O'Toole, S., \& Heber, U. 2006, A\&A, 452, 579
Perlmutter, S., Aldering, G., Goldhaber, G., et al. 1999, ApJ, 517, 565 Przybilla, N. 2005, A\&A, 443, 293

Przybilla, N., \& Butler, K. 2004, ApJ, 609, 1181

Przybilla, N., Nieva, M. F., \& Edelmann, H. 2006, in Proc. of the Second Meeting on Hot Subdwarf Stars, Baltic Astron., 15, 107

Riess, A. G., Fillipenko, A. V., Challis, P., et al. 1998, AJ, 116, 1009

Saffer, R. A., \& Liebert, J. 1995, in Proc. 9th European Workshop on White Dwarfs, ed. D. Koester, \& K. Werner (Springer Verlag), 221

Stehlé, C., \& Hutcheon, R. 1999, A\&AS, 140, 93 (SH)

Stobie, R. S., Kawaler, S. D., Kilkenny, D., O’Donoghue, D., \& Koen, C. 1997, MNRAS, 285, 651

Telting, J., \& Østensen, R. 2004, A\&A, 419, 685

Vidal, C. R., Cooper, J., \& Smith, E. W. 1973, ApJS, 25, 37 (VCS)

Vogt, S. S., et al. 1994, Proc. SPIE, 2198, 362

Whelan, J., \& Iben, I. Jr. 1973, ApJ, 186, 1007

Wilson, R. E., \& Devinney, E. J. 1971, ApJ, 166, 605

Woolf, V. M., Jeffery, C. S., \& Pollacco, D. L. 2002, MNRAS, 332, 34 